\title{
Bicuspid Aortic Valve
}

National Cancer Institute

\section{Source}

National Cancer Institute. Bicuspid Aortic Valve. NCI Thesaurus. Code C128803.

A cong enital anomaly in which the aortic valve has two leaflets. It is associated with mutations in the NOTCH1 gene or the SMAD6 gene, encoding neurogenic locus notch homolog protein 1 and mothers against decapentaplegic homolog 6, respectively. It is a clinically heterogeneous condition, with a high incidence of aortic valve and ascending aortic complications requiring surgery. 